\title{
Genetic Differences in the Development of Physical Dependence on Pentobarbital in Four Inbred Strains of Rats
}

\author{
Tsutomu SUZUKI 1,2,4, Yoko KOIKE1, Saizo YANAURA', \\ Frank R. GEORGE ${ }^{3,4}$ and Richard A. MEISCH ${ }^{2}$ \\ 'Department of Pharmacology. School of Pharmacy. Hoshi University, \\ Shinagawa-ku, Tokyo 142, Japan \\ ${ }^{2}$ Department of Psychiatry and Pharmacology, Medical School. \\ University of Minnesota, Minneapolis, MN 55455, U.S.A. \\ ${ }^{3}$ Department of Pharmacology and Toxicology, School of Pharmacy, \\ University of Maryland, Baitimore, MD 21201, U.S.A. \\ ${ }^{4}$ Behavior Genetics Laboratory, Preclinical Pharmacology Branch, \\ National Institute on Drug Abuse, Addiction Research Center. \\ Baltimore, MD 21224, U.S.A.
}

Accepted August 14, 1987

\begin{abstract}
Fischer 344 (F344), Lewis (LEW), spontaneously hypertensive (SHR) and Wistar Kyoto (WKY) rats were chronically fed food containing pentobarbital on an escalating drug dosage schedule over a period of 47 days. During treatment, the growth curve in LEW. SHR and WKY rats was suppressed as compared with the respective controls. Motor incoordination was evaluated by a rotarod performance test. The ranking of the motor incoordination was as follows: WKY $\gg$ LEW $>S H R>$ F344. After substitution of normal food for the pentobarbitaladmixed food, various signs of pentobarbital withdrawal occurred. The withdrawal signs from pentobarbital in F344, LEW and SHR rats were mild as compared with those in WKY rats. The order of the severity of withdrawal signs in four inbred strains was parallel to that for motor incoordination. These results suggest that the differences between strains in withdrawal can be attributed to differences in the degree of chronic CNS depression produced by pentobarbital.
\end{abstract}

Genetic differences in tolerance and physical dependence to several drugs have been reported. Among the more commonly used inbred strains of mice, the C57BL/6 strain seems to develop a greater degree of physical dependence on morphine (sensitivity to naloxone-induced jumping) than the DBA/2 strains (1). There have been several genetic studies of tolerance to and physical dependence on ethanol in inbred mice. Grieve et al. (2) reported that the C57BL strain developed tolerance much faster than does the DBA strain, and the C57BL strain showed brief and mild withdrawal signs in comparison

Address correspondence to: Dr. Tsutomu Suzuki, Department of Applied Pharmacology, School of Pharmacy, Hoshi University, 2-4-41, Ebara, Shinagawa-ku. Tokyo 142, Japan with the DBA strain. Goldstein and her collaborator $(3,4)$ showed a differential resistance to withdrawal seizures in the C57BL strain. These experiments suggest that genotype is an important determinant of the degree of tolerance to and physical dependence on ethanol and morphine.

Acute effects of barbiturates have also been examined in genetic studies. C57BL mice are known to be more sensitive than DBA and BALB mice to pentobarbital (5-7). Recently, Khanna et al. (8) reported that pentobarbital and barbital sleep times for spontaneously hypertensive (SHR) rats were significantly less than for Wistar Kyoto (WKY) rats, and barbiturate blood levels at awakening were significantly higher in SHR than in WKY rats. Very few studies of physical dependence on barbiturates have examined genetic 
factors, but it has been demonstrated that DBA mice show a markedly greater susceptibility to development of physical dependence on barbiturates than do C57BL mice $(5,9)$.

Crossland and Leonard (10) were the first to show that withdrawal from barbital can result in convulsions in rats. Other studies extended this finding to demonstrate that physical dependence on barbiturates can be demonstrated in rodents $(9,11-14)$. Until recently, severe physical dependence on pentobarbital as manifested by withdrawal convulsions had not been shown in rodents. However, reports from several laboratories have now documented the development of marked physical dependence on pentobarbital (15-18)

The purpose of the present study was to systematically investigate physical dependence on pentobarbital in four inbred strains of rats, the F344, LEW, SHR and WKY, and to investigate the possible relationship between degree of motor incoordination during pentobarbital treatment and severity of withdrawal. A procedure was used that can produce severe physical dependence (17).

\section{Materials and Methods}

Subjects: Fischer 344 (F344), Lewis (LEW), spontaneously hypertensive (SHR) and Wistar Kyoto (WKY) female rats were obtained from Charles River Japan, Inc. (Kanagawa. Japan) at 5 weeks of age. Animals were then housed in individual cages under a 12-hr light-dark cycle with food and water continuously available. The room temperature was maintained at $22 \pm 1^{\circ} \mathrm{C}$, and the relative humidity was maintained at $55 \pm 5 \%$. The rats were allowed to adapt to their environment for a period of 1 week.

Drug treatment: For preparing the drugadmixed food, sodium pentobarbital (Tokyokasei Kogyo. Tokyo, Japan) was mixed with a normal powdered food (CA-1, Japan Clea, Tokyo, Japan) in a mortar $(13,14)$. Each rat was allowed to eat the pentobarbitaladmixed food and to drink tap water ad libitum. Rats of each strain were divided into two groups, the pentobarbital-treated group $(n=7)$ and the control (non-treated) group $(n=6)$. The rats were chronically treated with pentobarbital according to the method of Suzuki et al. (17). The concentration of pentobarbital in the food was gradually increased, as shown in Table 1 . When rats were treated with one dose of pentobarbital. there was one food container in a cage. When rats were treated with two doses (e.g., 10 and $12 \mathrm{mg} / \mathrm{g}$ of food), there were two food containers with each dose in a cage. During several phases of training rats were given simultaneous access to two concentrations of pentobarbital in order to avoid or minimize toxicity of the drug. The body weight of rats and food consumption were measured daily at 14:30 hours. The daily pentobarbital intake was calculated as follows:

$$
\begin{aligned}
& \begin{array}{l}
\text { pentobarbital intake } \\
(\mathrm{mg} / \mathrm{kg} / \text { day })
\end{array} \\
& \text { food intake } \times \text { concentration } \\
& \frac{(\mathrm{g} / \text { day }) \quad(\mathrm{mg} / \mathrm{g} \text { of food })}{\text { body weight }(\mathrm{kg})}
\end{aligned}
$$

Table 1. Progressively increasing dosage schedule for development of physical dependence on pentobarbital in four inbred strains of rats

\begin{tabular}{cc}
$\begin{array}{c}\text { Pentobarbital concentration } \\
\text { (mg/g of food) }\end{array}$ & $\begin{array}{c}\text { Duration } \\
\text { (days) }\end{array}$ \\
\hline 1 & 3 \\
2 & 3 \\
4 & 3 \\
6 & 3 \\
8 & 3 \\
$10 \& 12$ & 6 \\
$12 \& 14$ & 6 \\
$14 \& 16$ & 10 \\
16 & 10 \\
\hline
\end{tabular}


Measurement of motor incoordination: Motor incoordination in pentobarbital-treated rats $w a s$ measured by the rotarod performance test $19 \mathrm{~cm}$ in diameter, $5.3 \mathrm{rom}, 5 \mathrm{~min}$ as standard: Natsume Seisakusho Co. Tokyo, Japan). Each rat was trained to run on a rotarod until it could remain there for $5 \mathrm{~min}$ without falling. The rotarod performance test was carried out at intervals of 1 to 3 days during pentobarbital treatment.

Withdrawal: Withdrawal was conducted by substituting normal food for pentobarbitaladmixed food on the last day of the treatment at 14:00 hours. Body weight and food intake were measured every $3 \mathrm{hr}$ after the termination of drug access. Following termination of drug treatment, rats were continuously observed over a $15 \mathrm{hr}$ interval that began $3 \mathrm{hr}$ after the end of drug treatment. All instances of abnormal behavior were recorded. To quantify the intensity of physical dependence on pentobarbital, a rating score for withdrawal signs was used. We classified the withdrawal signs into three grades, mild (score 1). intermediate (score 2) and severe (score 3). This grading method was based on the method of Tagashira et al. with modifications (12). The criteria of these signs are as follows: vocalization: vocalization in response to the tactile stimuli

irritability: become wild in response to the tactile or auditory stimuli and/or restlessness

aggression: destructive or attacking behavior towords the observers in response to the tactile or auditory stimuli

fascicular-twitch: shaking or trembling of nape

tremor: shaking or trembling of the whole body convulsion: spontaneous clonic convuision

Statistical analysis: Analysis for the incidence of withdrawal signs was performed by the chi-square $(2 \times 2)$-test. All other analyses were carried out using Student's $t$-test.

\section{Results}

Changes in body weight and drug intake: Relative to control rats, weight gain in LEW. SHR and WKY of the pentobarbital-treated groups was gradually suppressed beginning at drug concentrations of $8 \mathrm{mg}$ (WKY) or
10 and $12 \mathrm{mg}$ (LEW and SHR) of pentobarbital/g of food. In three strains. LEW, SHR and WKY, rats from the pentobarbital group showed significantly less weight gain than their respective control rats during the final pentobarbital concentration (LEW: $t=4.84, P<0.002$; SHR: $t=4.78, P<0.002$; WKY: $t=6.83, P<0.001$ ). However, F344 pentobarbital group rats grew significantly more than their respective controls $(t=2.53$, $P<0.05)$. Percent of growth suppression in WKY LEW. SHR and F344 as compared with their respective controls during the final pentobarbital concentration were 71.587 .6 . 88.9 and $108.4 \%$, respectively. Thus, the order of the degree of growth suppression in these four inbred strains of rats was: WKY $\geqslant L E W>$ $S H R>F 344$. Figure 1 presents pentobarbital intake during treatment. The range of pentobarbital intake in the four strains of rats was about 100 to $1200 \mathrm{mg} / \mathrm{kg} /$ day (1 to $16 \mathrm{mg} / \mathrm{g}$ of food). The mean drug intakes during the final pentobarbital concentration $(16 \mathrm{mg} / \mathrm{g}$ of food) in F344. LEW. SHR and WKY rats were 1070.8, 1094.6, 1180.4 and $992.0 \mathrm{mg} /$ $\mathrm{kg} /$ day, respectively. There was no significant difference in the final drug intake between the four strains.

Motor incoordination during pentobarbital treatment: Figure 2 presents changes in rotarod performance as a function of increases in pentobarbital concentration from $1 \mathrm{mg} / \mathrm{g}$ to $16 \mathrm{mg} / \mathrm{g}$ of food. The fifty percent inhibition concentration for rotarod performance in WKY occurred at 10 and $12 \mathrm{mg}$ pentobarbital/g of food. The $50 \%$ inhibition concentration in LEW and SHR was at 12 and $14 \mathrm{mg}$ pentobarbital/g of food. However, rotarod performance in F344 was suppressed no more than $30 \%$ even at the pentobarbital concentration of $16 \mathrm{mg} / \mathrm{g}$ of food. The order of the degree of motor incoordination produced by pentobarbital in these four inbred strains of rats was: WKY $>L E W>S H R>$ F344. On the first or third day of presentation of each concentration, the rotarod performance was suppressed markedly; and across subsequent days, this suppression decreased gradually in most strains. These results indicate that all four strains showed development of tolerance to pentobarbital.

Withdrawal signs: After substituting normal 
Pentobarbital concentration (mg/g of food)

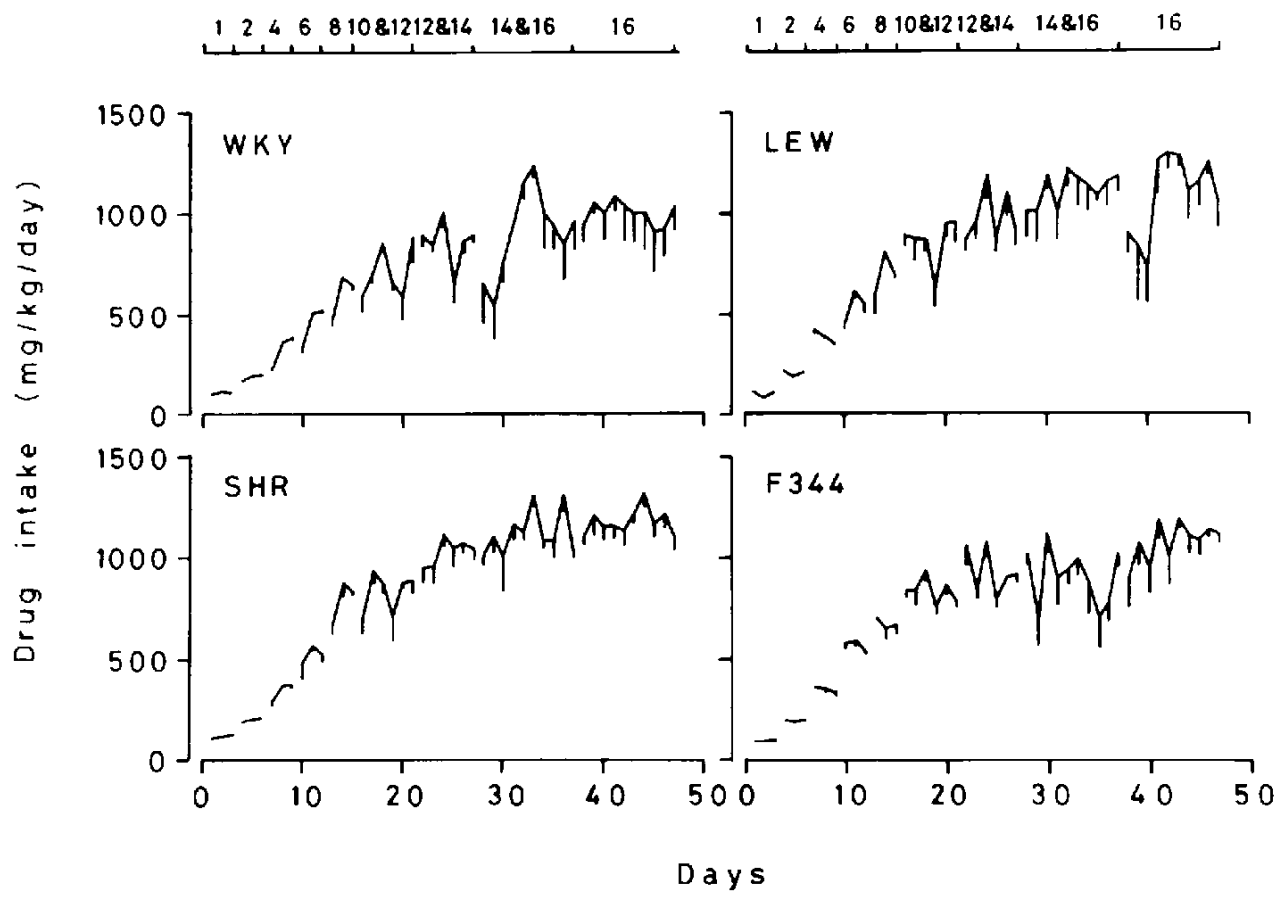

Fig. 1. Changes in mean daily pentobarbital intake $(\mathrm{mg} / \mathrm{kg} /$ day) during pentobarbital-admixed food treatment on a schedule of gradually increasing dosages in four inbred strains of rats. Each point is a mean of 7 observations, and the lines indicate S.E.M.

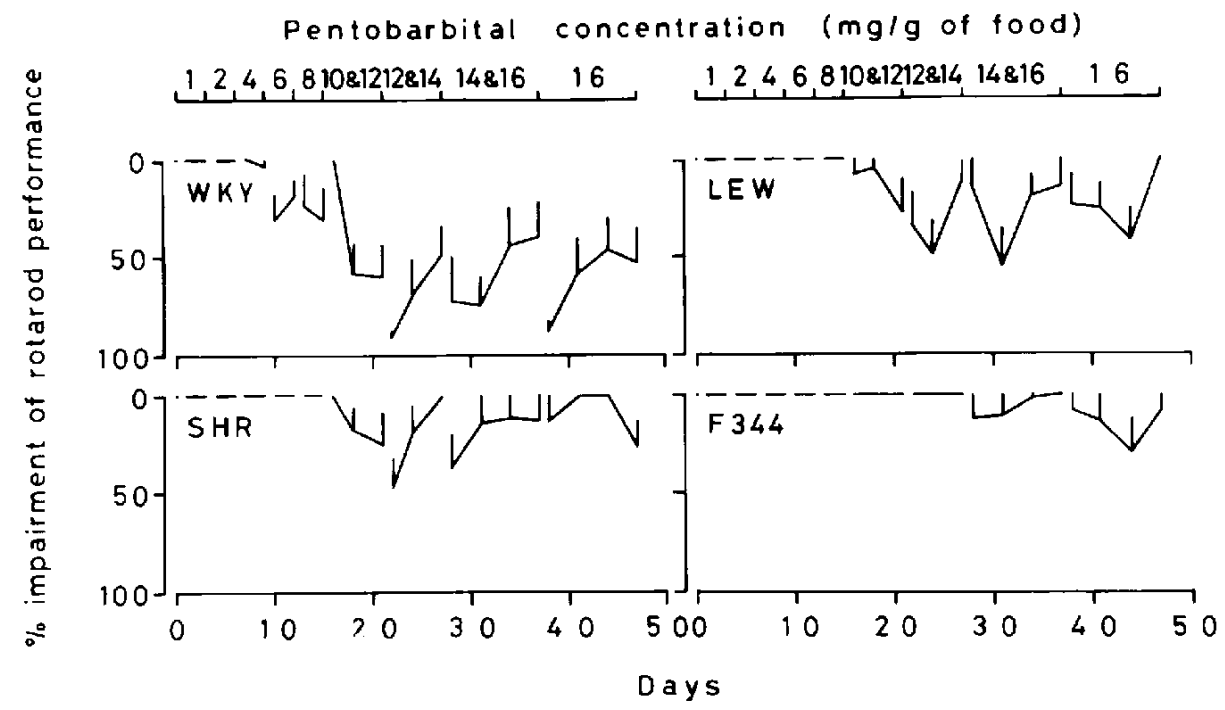

Fig. 2. Alterations in rotarod performance during pentobarbital-admixed food treatment in four inbred strains of rats. Each point is a mean of 7 observations, and the lines indicate S.E.M. 
Table 2. Behavioral changes after the termination of pentobarbital treatment in four inbred strains of rats

\begin{tabular}{|c|c|c|c|c|}
\hline & \multicolumn{4}{|c|}{ Positive animals/Total number of animals } \\
\hline & WKY & LEW & SHR & F344 \\
\hline \multicolumn{5}{|l|}{ Weight loss } \\
\hline $5-10 \%(1)$ & $1 / 7$ & $2 / 7$ & $2 / 7$ & $5 / 7$ \\
\hline $10-15 \%(2)$ & $1 / 7$ & $2 / 7$ & $2 / 7$ & $1 / 7$ \\
\hline $15 \%<(3)$ & $2 / 7$ & $0 / 7$ & $0 / 7$ & $0 / 7$ \\
\hline Vocalization (2) & $7 / 7$ & $3 / 7$ & $3 / 7$ & $0 / 7^{* *}$ \\
\hline Irritability (2) & $6 / 7$ & $0 / 7^{* *}$ & $1 / 7^{*}$ & $0 / 7^{* *}$ \\
\hline Aggression (2) & $4 / 7$ & $0 / 7$ & $0 / 7$ & $0 / 7$ \\
\hline Muscle rigidity (2) & $7 / 7$ & $6 / 7$ & $4 / 7$ & $2 / 7^{*}$ \\
\hline Straub's tail (2) & $7 / 7$ & $7 / 7$ & $7 / 7$ & $7 / 7$ \\
\hline Ear-twitching (2) & $7 / 7$ & $7 / 7$ & $5 / 7$ & $4 / 7$ \\
\hline Fascicular-twitch (3) & $7 / 7$ & $1 / 7^{* *}$ & $1 / 7^{* *}$ & $0 / 7^{* *}$ \\
\hline Tremor (3) & $6 / 7$ & $0 / 7^{* *}$ & $0 / 7^{* *}$ & $0 / 7^{* *}$ \\
\hline Convulsion (3) & $5 / 7$ & $0 / 7^{*}$ & $0 / 7^{*}$ & $0 / 7^{*}$ \\
\hline Death (3) & $1 / 7$ & $0 / 7$ & $0 / 7$ & $0 / 7$ \\
\hline Withdrawal scores & $20.3 \pm 1.6$ & $7.9 \pm 0.9 b$ & $7.0 \pm 1.0^{\mathrm{b}}$ & $4.6 \pm 0.8^{a}$ \\
\hline
\end{tabular}

${ }^{*} \mathrm{P}<0.05$ vs. WKY, * $\mathrm{P}<0.01$ vs. WKY. The chi-square $(2 \times 2)$ test was used in the statistical evaluation for each of the withdrawal signs. aP<0.05 vs. LEW, ${ }^{b} P<0.001$ vs. WKY. Analysis for the withdrawal scores was performed by Student's t-test. The number in parenthesis indicates the rating score for withdrawal signs. The withdrawal scores calculated by the sum of the rating score per animal.

food for pentobarbital-admixed food, several signs of pentobarbital withdrawal were observed 3 to $72 \mathrm{hr}$ later. These signs included vocalization, irritability, aggression, muscle rigidity, Straub's tail, ear-twitching. tremor and convulsions (Table 2). The incidences of irritability, fascicular-twitch, tremor and convulsions in WKY were significantly higher than those in F344, LEW and SHR rats. Furthermore, there were significant differences between the WKY and F344 rats in the occurrence rate of vocalization and muscle rigidity. Withdrawal scores in WKY was significantly greater than that in LEW. SHR $(P<0.05)$ and $F 344$ rats $(P<0.001)$; and there was significant difference between the LEW and F344 rats in the withdrawal scores. One out of 7 WKY rats died of convulsions 7 $\mathrm{hr}$ after the end of drug access. The order of the degree of withdrawal signs in these four inbred strains of rats was: WKY $\gg$ LEW $>$ $\mathrm{SHR}>\mathrm{F} 344$. As shown in Fig. 3, body weights of animals which were treated with pentobarbital decreased immediately after pentobarbital withdrawal. Body weights of pentobarbital-treated groups and the respective control in each inbred strain at the

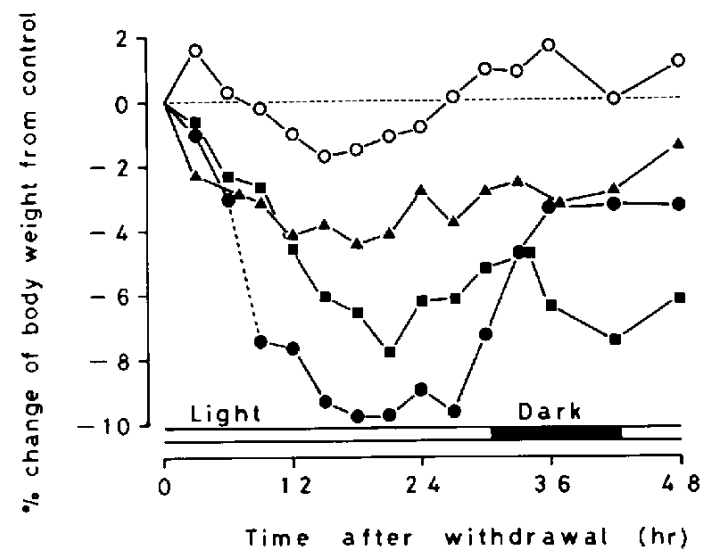

Fig. 3. Time course changes in body weight (\%) from the respective control after the termination of pentobarbital treatment in four inbred strains of rats (WKY: O, F344: $\square$, LEW: A, SHR: O). The dotted lines represent the respective controls. The asterisk indicates that a rat died of convulsions $7 \mathrm{hr}$ after the end of drug access. Each point is a mean of 6 to 7 observations.

beginning of pentobarbital withdrawal were 145.0 and $207.3 \mathrm{~g}$ in WKY, 179.4 and $163.7 \mathrm{~g}$ in F344, 185.3 and $209.0 \mathrm{~g}$ in LEW and 179.9 and $204.8 \mathrm{~g}$ in SHR rats. The magnitudes of 
maximum weight loss after withdrawal in WKY, F344, LEW and SHR rats were 9.74. 7.77, 4.44 and 1.69, and the areas under the curve were ( $48 \mathrm{hr}$ total) $-300.62,-248.79$. -145.64 and +3.45 , respectively.

\section{Discussion}

The present experiments demonstrate that pentobarbital withdrawal severity is strongly influenced by genetic factors. WKY rats had the lowest drug intake, but showed the greatest intoxication and most severe physical dependence on pentobarbital. In contrast the withdrawa! signs exhibited by F344. LEW and SHR rats were quite mild.

In this study, physical dependence on pentobarbital was established with a drugadmixed food method (12-14, 19), which has been successfully used in the past for the development of physical dependence on pentobarbital in Sprague-Dawley rats (16. 17). The drug dosage schedule used in the present study was the same as that used in an earlier study (17). Thus, the withdrawal syndrome previously observed in SpragueDawley rats can be compared to the syndromes seen in this study. The pentobarbital withdrawal signs in SpragueDawley rats were comparable to those in WKY rats but more severe than those in F344, LEW and SHR rats.

An important finding of the present study is that the rank order of severity of withdrawal was the same as for chronic impairment of rotarod performance (i.e., WKY $\gg L E W>S H R$ $>$ F344). There was a significant correlation between the withdrawal scores and the area under the curve of impairment of rotarod performance $(r=0.994)$. It is possible that the degree of impairment is causally related to the degree of chronic CNS depression produced by pentobarbital. The correlation between severity of withdrawal and extent of chronic CNS depression is consistent with previous studies where the degree of depression was manipulated by using different barbiturate or alcohol doses or by giving the drug for different durations $(17,20-22)$.

On the other hand, Okamoto et al. (21) demonstrated individual variations in the drug elimination half-lives related significantly to the severity of physical dependence produced by pentobarbital: the longer halflife, the more intense the production of physical dependence by pentobarbital. However, blood pentobarbital disappearance curves after a test dose of pentobarbital showed no apparent difference between SHR and WKY rats with respect to either the halflife of pentobarbital disappearance or the apparent volume of distribution (8). These results suggest that the differences between the SHR and WKY rats in severity of withdrawal are not related to differences in elimination rates.

In the present study, the strain differences in pentobarbital withdrawal intensities may reflect either differences in CNS sensitivities or differences in drug metabolism or disposition. In two studies, acute administration of pentobarbital resulted in greater sleep times for the WKY rats than for the SHR rats (8, 23). Khanna et al. (8) reported that barbiturate blood levels at awakening were significantly higher in SHR than in WKY rats; and thus, the difference in sleep times could be attributed to differential CNS sensitivity to pentobarbital. In contrast, Willis and Queener (23) reported that the blood levels of $\left({ }^{14} \mathrm{C}\right)$ pentobarbital at awakening in SHR rats were not significantly different from WKY rats. Their interpretation was that the difference in sleep time was due to differences in drug metabolism or disposition. In subsequent studies, we have found differences in pentobarbital sleep time for the four strains of inbred rats (WKY. SHR, LEW and F344), but the mean concentrations of pentobarbital in serum and brain at awakening were not significantly different among these strains ( $T$. Suzuki et al., unpublished data). Thus, our results support the findings of Willis and Queener (23).

After the withdrawal of pentobarbital, F344 rats showed marked losses in body weight, but other withdrawal signs were very mild. Other results obtained in this study suggest that this is due to the significant increase. relative to the control, in the body weight of the F344 pentobarbital rats prior to withdrawal. It is known that in rats, chronic administration of barbiturates can produce polyphagia and increases in body weight $(12$, 24) 
The results of this study emphasize the important role of genetic factors as determinants of both response to chronic drug treatment and withdrawal severity. Our findings extend results reported in mice to another species, namely rats, and also extend previous findings by relating withdrawal severity to the degree of chronic motor impairment, as opposed to the degree of acute motor impairment. In this study, motor impairment resulting from chronic pentobarbital treatment was rank order correlated with withdrawal severity from the same drug. Our data and other related data using these rat strains suggest that motor impairment after chronic pentobarbital treatment has in common at least some of the biochemical substrates which determine development and severity of tolerance and physical dependence on pentobarbital.

Acknowledgments: We wish to thank K. Takahashi for his assistance. This research was supported by an Ohtani Research Grant, Japan to T. Suzuki, by a grant from the University of Minnesota's Office of Alcohol and Other Drug Abuse Programming to T. Suzuki and R.A. Meisch, by New Investigator Research Award AA-06104 and AA-06924 to F.R. George from the National Institute on Alcohol Abuse and Alcoholism. U.S.A., and by grant DA-00944 to R.A. Meisch from the National institute on Drug Abuse. U.S.A.

\section{References}

1 Brase, D.A., Loh, H.H. and Way, E.L.: Comparison of the effects of morphine on locomotor activity, analgesia and primary and protracted physical dependence in six mouse strains. J. Pharmacol. Exp. Ther. 201, 368-374 (1977)

2 Grieve, S.J., Griffiths, P.J. and Littleton, J.M.: Genetic influences on the rate of development of ethanol tolerance and the ethanol physical withdrawal syndrome in mice. Drug Alcohol Depend. 4, 77-86 (1979)

3 Goldstein, D.B : Inherited differences in intensity of alcoho! withdrawal reactions in mice. Nature 245, 154-156 (1973)

4 Goldstein, D.B. and Kakihana, R.: Alcohol withdrawal reactions and reserpine effects in inbred strains of mice. Life Sci. 15, 415-425 (1974)

5 Nabeshima, T. and Ho, I.K.: Pharmacological responses to pentobarbital in different strains of mice. J. Pharmacol. Exp. Ther 216, 198-204 (1981)
6 Randall, C.L. and Lester, D.: Differential effects of ethanol and pentobarbital on sleep time in C57BL and BALB mice. J. Pharmacol. Exp. Ther. 188, 27-33 (1974)

7 Siemens, A.J. and Chan, A.W.K.: Differential effects of pentobarbital and ethanol in mice. Life Sci. 19, 581-590 (1976)

8 Khanna, J.M., Le, A.D., Kalant, H. and Kim, C.: Differential sensitivity to ethanol, pentobarbital, and barbital in spontaneously hypertensive and normotensive Wistar-Kyoto rats. Psychopharmacology (Berlin) 86, 296-301 (1985)

9 Belknap, J.K., Wadingham, S. and Ondrusek, G.: Barbiturate dependence in mice induced by a simple short-term oral procedure. Physiol. Psychol. 1. 394--396 (1973)

10 Crossland, J. and Leonard, B.E.: Barbiturate withdrawal convulsions in the rat. Biochem. Pharmacol. Supp. 12, 103 (1963)

11 Flint, B.A. and Ho, I.K.: Continuous administration of barbital by pellet implantation. J. Pharmacol. Methods 4, 127-139 (1980)

12 Tagashira, E., Izumi, T. and Yanaura, S.: Experimental barbiturate dependence. I. Barbiturate dependence development in rats by drugadmixed food (DAF) method. Psychopharmacology (Berlin) 57, 137-144 (1978)

13 Yanaura, S., Suzuki, T. and Tagashira, E.: Study on drug dependence in rats.-Substitution test and time course of body weight--. Folia Pharmacol. Japon. 70, 649-658 (1974) (Abs. in English)

14 Yanaura, S., Tagashira, E. and Suzuki, T.: Physical dependence on morphine, phenobarbital and diazepam in rats by drug-admixed food ingestion. Japan. J. Pharmacol. 25, 453-463 (1975)

15 Ho, I.K., Yamamoto, I. and Loh, H.H.: A model for the rapid development of dispositional and functional tolerance to barbiturates. Eur. J. Pharmacol. 30, 164--171 (1975)

16 Martin, W.R., McNicholas, L.F. and Cherian, S.: Diazepam and peritobarbital dependence in the rat. Life Sci. 31, 721-730 (1982)

17 Suzuki, T., Koike, Y.. Yoshii, T. and Yanaura, S.: Sex differences in the induction of physical dependence on pentobarbital in the rat. Japan. J. Pharmacol. 39, 453-459 (1985)

18 Yutrzenka, G.J., Patrick, G.A. and Rosenberge, W.: Continuous intraperitoneal infusion of pentobarbital: A model of barbiturate dependence in the rat. J. Pharmacol. Exp. Ther. 232, 111-118 (1985)

19 Suzuki, T., Fukumori, R., Yoshii, T., Yanaura, S., Satoh, T. and Kitagawa, H.: Effect of $p$ chlorophenylalanine on diazepam withdrawal 
signs in rats. Psychopharmacology (Berlin) 71, 91-93 (1980)

20 Goldstein. D.B.: Relationship of alcohol dose to intensity of withdrawal signs in mice. J. Pharmacol. Exp. Ther. 180, 203-215 (1972)

21 Okamoto, M., Himman, D. and Aaeronson, L.M.: Comparison of ethanol and barbiturate physical dependence. J. Pharmacol. Exp. Ther. 218, 701-708 (1981)

22 Seevers, M.H. and Deneau, G.A.: Physiological aspects of tolerance and physical dependence.
In Physiological Pharmacology, Edited by Hoffman, F.G, and Root, W.S., p. 565-640. Academic Press, New York (1984)

23 Willis, L.R. and Queener, S.F.: Pentobarbital sleeping time and waking blood levels in spontaneously hypertensive rats. Can. J. Physiol. Pharmacol. 55, 1205-1207 (1977)

24 Leonard, B.E.: The effect of the chronic administration of barbitone sodium on pituitary-adrenal function in the rat. Biochem. Pharmacol. 15, 263-268 (1966) 